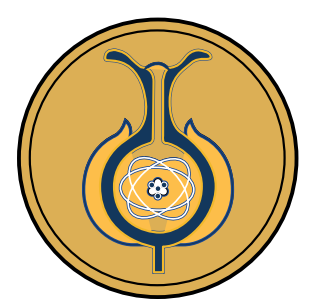

\title{
El conocimiento matemático del profesor acerca de la parábola: diseño de un instrumento para investigación
}

\author{
Teachers' mathematical knowledge of parabolas: Designing an instrument \\ for research
}

O conhecimento matemático do professor sobre a parábola: desenho de um instrumento para investigação

Elizabeth Advíncula Clemente ${ }^{1} \bullet$ Marisel Beteta Salas $^{1} \bullet J_{0}$ Cé Carlos León Ríos ${ }^{1} \bullet$ Isabel Torres Céspedes ${ }^{1} \cdot$ Miguel Montes $^{2}$

Received: Jan/11/2020 • Accepted: May/12/2020 • Published: Jan/31/2021.

\section{Resumen}

La investigación en Educación Matemática suele centrarse en compartir los resultados de la aplicación de instrumentos de obtención de información. Si bien dichos instrumentos suelen describirse en la sección de metodología, el proceso de su desarrollo no suele ser muy transparente para el lector. En esta investigación el objetivo es abordar dicha problemática y describir con todo detalle el proceso de generación de un cuestionario, basado en el modelo MTSK, para la exploración del conocimiento del profesor acerca de la parábola, entendida como cónica. Se presenta el diseño de un instrumento de carácter cualitativo que permite hacer una aproximación al conocimiento matemático del profesor acerca de la parábola, poniendo énfasis tanto en el uso de la teoría, como de la triangulación, interna y externa, como elementos clave en el diseño metodológico. El principal aporte fue determinar pautas para el diseño de cuestionarios basados en el modelo MTSK, las cuales pueden ser tomadas en cuenta al margen de los contenidos matemáticos.

Palabras clave: educación matemática; conocimiento matemático; funciones; parábola, cuestionario, MTSK, conocimiento del profesor.

\section{Abstract}

Research in mathematics education usually focuses on sharing results from the application of instruments to obtain data. Even though these instruments are usually described in the methodology section, their development process tends to not be transparent for the reader. The objective of this paper is to study this problem and describe in detail the process of creating a questionnaire based on the MTSK model, in order to explore the teacher's knowledge of parabolas, understood as conic sections. We present the design of

Elizabeth Advíncula Clemente, $\$ eadvincu@ulima.edu.pe, (D https://orcid.org/0000-0003-3941-3139

Marisel Beteta Salas, $\triangle$ mbeteta@ulima.edu.pe, (1) https://orcid.org/0000-0003-0100-8517

José Carlos León Ríos, \jleonr@ulima.edu.pe, (1) https://orcid.org/0000-0002-3128-788X

Isabel Torres Céspedes, $、$ iztorres@ulima.edu.pe, (D https://orcid.org/0000-0002-0673-8984

Miguel Montes, \ miguel.montes@ddcc.uhu.es, (D https://orcid.org/0000-0003-3181-0797

1 Instituto de Investigación Científica, Universidad de Lima, Lima, Perú

2 Centro de Investigación COIDESO de la Universidad de Huelva, Huelva, España 
a qualitative instrument that allows for the approximation of the teacher's mathematical knowledge of parabolas, emphasizing on the use of theory, as well as both internal and external triangulation, as key elements in the methodological design. The main contribution of this paper is to give guidelines for the design of questionnaires based on the MTSK model, which can be taken into account regardless of the mathematical contents.

Keywords: Mathematics education; mathematical knowledge; functions; parabola, questionnaire, MTSK, teacher's knowledge.

\section{Resumo}

A pesquisa na Educação Matemática tende a estar centrada no compartilhamento dos resultados da aplicação de instrumentos de alcance de informação. Embora tais instrumentos se inclinem a estar descritos na seção de metodologia, o processo de seu desenvolvimento tende a não ser muito transparente para o leitor. Nesta pesquisa o objetivo é abordar essa problemática e descrever detalhadamente o processo de criação de um questionário, com base no modelo MTSK, para a exploração do conhecimento do professor sobre a parábola, entendida como cônica. É apresentado o desenho de um instrumento de carácter qualitativo, que permite fazer uma aproximação ao conhecimento matemático do professor sobre a parábola, colocando ênfase tanto no uso da teoria quanto da triangulação, interna e externa, como elementos-chave no desenho metodológico. A principal contribuição foi determinar pautas para o desenho de questionários baseados no modelo MTSK, que podem ser levadas em consideração independentemente dos conteúdos matemáticos.

Palavras-chave: educação matemática; conhecimento matemático; funções; parábola, questionário, MTSK, conhecimento do professor.

\section{INTRODUCCIÓN}

Las investigaciones sobre el conocimiento del profesor han tenido diferentes focos en los últimos años, uno de los principales es la evaluación del conocimiento matemático, fundamentalmente de los profesores de Educación Primaria (e.g. Baumbert y Kunter, 2013; Hill, Ball y Schilling, 2008). Pero, en recientes foros internacionales (e.g. ICME 13, PME 43) se ha puesto de relieve el estudio del conocimiento del profesor de Educación Secundaria.

En trabajos desarrollados desde el MTSK (Carrillo-Yañes et al., 2018), se han hecho acercamientos, tanto al conocimiento del profesor sobre álgebra (Guerrero, Flores-Medrano y Carrillo, 2015), como al de las funciones (Espinoza-Vázquez, Zakaryan y Carrillo, 2018). Sin embargo, tras realizar, entre mediados de 2018 y 2019, una búsqueda bibliográfica en repositorios de tesis doctorales, así como en revistas internacionales especializadas en educación matemática, no hemos identificado investigaciones ligadas al conocimiento profesional acerca de la parábola, interés central de este artículo. Asimismo, identificamos la problemática de la no sistematización en el diseño de herramientas de recojo de información en estudios ligados a la exploración del conocimiento de profesores.

Entendemos que la investigación cualitativa suele tener un carácter abierto en las dinámicas de obtención de datos, al contemplar observaciones de aula (participantes o no), y entrevistas habitualmente (semi) estructuradas y (semi)abiertas. Sin embargo, en las investigaciones revisadas, 
la descripción de cómo se desarrollan las herramientas de investigación se limita a una descripción de los dominios o subdominios de conocimiento de los que se pretende obtener información. En Carreño y Climent (2019), se establece:

Se propone completar un cuestionario de respuesta abierta, compuesto por cuatro situaciones de enseñanza, tres de las cuales abordan la identificación de aspectos determinantes en la concepción de distintos cuadriláteros, las características necesarias para definirlos y la clasificación jerárquica de los mismos. ... la Situación 3 ... indaga, a priori el conocimiento matemático base incluido en el KoT y el conocimiento de la definición como práctica matemática (KPM), en un contexto de enseñanza que precisa de los futuros profesores el reparo en el conocimiento matemático de los hipotéticos estudiantes involucrados en la situación dada (KFLM). (pp. 33-34)

Si bien entendemos que cualquier trabajo de investigación tiene la necesaria acotación derivada de las estructuras habituales de publicación, nuestro interés se centra en los detalles del diseño de herramientas de obtención de información, especialmente en los cuestionarios. Así, la descripción anterior no nos permite profundizar en los detalles de las decisiones tomadas por los investigadores al definir su instrumento de obtención de información.

En esta investigación tenemos como objetivo abordar dicha limitación y explicitar, con el máximo detalle posible, el proceso de elaboración de un cuestionario, basado en el modelo MTSK, para explorar el conocimiento matemático de profesores de secundaria peruanos acerca de la parábola. Más allá del detalle del cuestionario cuyo diseño mostraremos, daremos pautas para el diseño de cuestionarios basados en el modelo MTSK.

\section{REFERENTES TEÓRICOS}

Esta investigación tiene dos referentes principales en cuanto a su fundamentación. Por un lado, el modelo de conocimiento especializado del profesor de matemáticas supone el referente para acercarse a la estructuración de los elementos de conocimiento profesional que se pretenden explorar en el cuestionario. Por otro lado, los elementos referidos a la comprensión de la parábola aportan la concreción, respecto del contenido matemático que nos interesa aquí, los cuales permitirán dar contenido al propio cuestionario.

\section{Conocimiento profesional: MTSK}

En este trabajo usamos el modelo de conocimiento especializado del profesor de matemáticas-MTSK-(Carrillo et al., 2018) para diseñar un cuestionario, como herramienta analítica que permite profundizar en el conocimiento del profesor, cuyos inicios se basan en los dominios del conocimiento de la materia y el conocimiento didáctico del contenido propuestos por Shulman (1986). El MTSK tiene una visión intrínseca de la noción de especialización (Scheiner, Montes, Godino, Carrillo y Pino-Fan, 2019), que asume que el conocimiento del profesor es especializado siempre que sea útil para la profesión docente. Así, el MTSK se constituye como un modelo que permite analizar el conocimiento que el profesor pone en juego en cualquier tarea relacionada con la docencia, como la preparación de clases, la discusión con otros docentes, la enseñanza en aula o la reflexión posterior. Propone dos dominios de conocimiento: conocimiento matemático (MK) y conocimiento didáctico 
del contenido (PCK), y un tercer dominio que abarca las creencias del profesor sobre la matemática, su enseñanza y su aprendizaje. En este trabajo nos centraremos en el dominio de conocimiento matemático, por lo que no describiremos los otros.

El dominio MK abarca el conocimiento matemático que el profesor usa, o puede usar, en cualquier actividad; el cual debe trascender al contenido matemático que se pretende que aprenda un estudiante del nivel en el que enseña, no solo en cantidad de conocimiento sino también en la naturaleza de este. El MK se descompone en tres subdominios: conocimiento de los temas (KoT), conocimiento de la estructura matemática (KSM), y conocimiento de la práctica matemática (KPM).

El KoT abarca lo que conoce el profesor respecto al tema que enseña, así como la forma en que lo conoce. Incluye el conocimiento de conceptos, definiciones, fenómenos, procedimientos, registros de representación, propiedades y sus fundamentos, que no solo pueden ser objeto de enseñanza-aprendizaje, sino que a su vez pueden definir nuevos entes matemáticos. Es así como un profesor puede conocer una o múltiples definiciones de un concepto matemático, así como uno o varios algoritmos asociados a un procedimiento.

El KSM abarca el conocimiento que el profesor posee sobre las conexiones entre contenidos matemáticos. Estas conexiones pueden darse entre elementos del mismo tema matemático o entre elementos de temas diferentes. Incluye cuatro tipos de conexiones: las de simplificación, las de complejización, las auxiliares, y las transversales. Las conexiones de simplificación y complejización hacen referencia a establecer relaciones de un contenido dado con contenidos curriculares previos o posteriores, útiles para su enseñanza. Las auxiliares vienen dadas por la introducción de un contenido matemático en un momento concreto, con un rol coadyuvante al trabajo matemático que se está realizando (e.g. el uso de la regla de Ruffini al calcular ceros de una derivada). Las conexiones transversales vienen dadas por la relación con elementos matemáticos transversales, como la proporcionalidad.

El KPM contempla el conocimiento matemático del profesor de naturaleza sintáctica, es decir, ligado a las reglas de construcción de un nuevo conocimiento matemático. Aquí, encontramos un conocimiento de diferentes formas de validación (Stylianides, Bieda y Morselli, 2016), como la demostración; y de elementos relacionados con la resolución de problemas, como las heurísticas y prácticas ligadas a la construcción de teoría (Bass, 2017).

Por otro lado, la exploración del conocimiento profesional ha tenido diversas aproximaciones metodológicas. Así, encontramos investigaciones que siguen enfoques cuantitativos y cualitativos. Los estudios cuantitativos, por lo general, pretenden evaluar el conocimiento de los profesores (e.g. Hill, Sleep, Lewis y Ball, 2007; Borko et al., 1992; Leinhardt y Smith, 1985), y en ellos se suelen usar cuestionarios y entrevistas. Estos cuestionarios se "basan en escenarios de enseñanza en el aula y están entrelazados con conocimiento del contenido" (Ball, 1990, p. 452). Encontramos estudios cualitativos en los que una técnica habitual de recojo de información es la observación en aula, la cual suele complementarse con entrevistas o cuestionarios. Para el tratamiento analítico de los datos se recurre al uso de instrumentos y procesos adaptados al tópico de estudio (Borko et al., 1992; Leinhardt y Smith, 1985; Hill et al., 2007), en busca de profundizar en el conocimiento matemático 
del profesor para la enseñanza, pues "la habilidad de los maestros no solo implica destreza para resolver problemas sino también para comprender el contenido de las formas necesarias para enseñarlo" (Hill, et al., 2007 p. 125). Las observaciones proporcionan oportunidades interesantes para acceder al conocimiento matemático que el profesor moviliza en acción (Flores-Medrano, Escudero y Aguilar, 2013).

El MTSK ha sido aplicado en diferentes niveles educativos (primaria, secundaria y superior), tanto con profesores en activo como en formación. Metodológicamente, desde su génesis se defiende que se requiere de una aproximación multimetodológica para profundizar en la complejidad del conocimiento del profesor. En general, los estudios desarrollados desde una perspectiva interpretativa hacen aproximaciones basadas en estudios de caso, usando observaciones de aula, entrevistas y cuestionarios (Carrillo y Contreras, 2017). En estudios que usan MTSK, los cuestionarios han sido usados tanto para la exploración del conocimiento profesional ligado a determinados temas (e.g. Carreño y Climent, 2019), como para recoger datos en investigaciones cuantitativas (e.g. Montes, Contreras, Liñán, Muñoz-Catalán, Climent y Carrillo, 2015). El MTSK sirve como herramienta teórica y analítica que permite identificar el conocimiento especializado del profesor de matemática y comprender su naturaleza, de manera sistemática y organizada para su análisis (Escudero, 2015).

\section{Parábola}

Aquí presentamos antecedentes relacionados con la cognición y el trabajo matemático sobre la parábola. Cabe destacar que los trabajos ubicados en repositorios y revistas especializadas en educación matemática, tras una búsqueda con la palabra clave 'parábola' y un análisis de los artículos resultantes de dicha búsqueda, no dan cuenta de referencias ligadas al conocimiento profesional de la parábola, sino más bien a reflexiones sobre estrategias para su enseñanza, de forma que se usarán resultados ligados a su aprendizaje.

En el contexto peruano, el Ministerio de Educación (MINEDU) es la entidad encargada de formular las políticas educativas, normar la gestión pedagógica y desarrollar estrategias que promuevan la capacitación de docentes. En el Currículo Nacional de la Educación Básica y el Programa Curricular de Educación Secundaria (MINEDU, 2016), se propone que los estudiantes deben ser capaces de resolver problemas relacionados con la parábola en diversos contextos, así como también el desarrollo de capacidades como modelar objetos con formas geométricas, comunicar su comprensión sobre las formas y sus relaciones geométricas, usar estrategias y procedimientos para orientarse en el espacio, y argumentar afirmaciones sobre relaciones geométricas. Sin embargo, Gómez y Ruiz (2014) hacen notar que el trabajo matemático sobre la parábola no se está desarrollando en toda su extensión en contextos peruanos, y aseguran la falta de resolución de problemas en contextos significativos como el de tiro parabólico o el de la luz proyectada por faros. Gómez y Ruiz (2014) también mencionan la necesidad de enriquecer las estrategias y recursos de los docentes haciendo uso de GeoGebra, de la proyección de un video o una calculadora científica, que implique una metodología innovadora en el aula.

Por otro lado, Del Río (2018) indica que uno de los principales problemas que tienen los estudiantes en el aprendizaje de la parábola es que la consideran un objeto 
matemático abstracto, que no tiene mucha utilidad en la vida humana y, por ello, le restan importancia. Es común observar que los estudiantes tienden a un aprendizaje memorístico de las ecuaciones, tienen dificultades para relacionar diversas expresiones algebraicas y no relacionan de forma lógica la representación algebraica con la geométrica (Gómez y Carulla, 2000). Precisamente, Just y Carpenter (1985) señalan que los estudiantes identifican las figuras cónicas en un contexto general, enunciándolas o visualizándolas en un esquema gráfico; pero al hacer un trabajo que involucre sus representaciones, características, propiedades y aplicaciones, no manifiestan diferencias en su interpretación ni reconocen las propiedades de sus elementos. Asimismo, estudiantes universitarios muestran concepciones equivocadas y errores frecuentes en la resolución de tareas con la parábola (López-Mesa, Aldana-Bermúdez y Alonso-Arboleda, 2013), posiblemente relacionados con el aprendizaje errado de este concepto desde el bachillerato, se da una definición institucional sin tener en cuenta los objetos personales (Godino y Batanero, 1994). También presentan dificultades cuando relacionan los gráficos con las fórmulas o ecuaciones, no identifican el eje de simetría de una parábola y otras cónicas (Ruiz, 2014).

Respecto al tratamiento de la parábola como una cónica, Santa y Jaramillo (2007) señalan que los estudiantes resuelven problemas apoyados en procesos algorítmicos y memorísticos, omitiendo la comprensión del concepto y evidenciando un desconocimiento de la relación existente entre sus elementos. Por ejemplo, si la parábola es rotada o traslada respecto a su posición inicial, los estudiantes encuentran dificultades para reconocer las propiedades que se mantienen invariantes. En este sentido, Lara (2016) señala que los estudiantes de educación básica tienen problemas con la comprensión de la parábola como lugar geométrico y considera que esto se debe, por un lado, al poco dominio que tienen los docentes acerca del concepto parábola; y por otro, al escaso conocimiento que tienen sobre el uso de recursos didácticos que favorecen su aprendizaje, como el uso de las TIC que facilitan una interactividad rápida y dinámica con el objeto matemático.

Por otro lado, el estudio de las cónicas desde la geometría analítica continúa dando mayor énfasis a los dominios algebraicos sobre los geométricos, enfocando el aprendizaje en la algoritmia y la memorización, donde los procesos algebraicos solapan las construcciones geométricas. Es común que, en contextos donde se trabajan las cónicas desde la geometría analítica, la exigencia a los alumnos se centre en el dominio de las expresiones algebraicas de las cónicas. Sin embargo, su función en la actividad matemática es más ilustrativa; es decir, no constituye un contexto de interacción con la naturaleza geométrica de la cónica.

Asimismo, la evaluación censal de estudiantes y la evaluación muestral de estudiantes de Perú, realizadas en octubre y noviembre de 2018 (MINEDU, 2016), muestran que existe una caída pronunciada en los resultados, donde la proporción de alumnos con rendimiento satisfactorio en matemáticas bajó del $37,4 \%$ al $14,6 \%$ en colegios públicos, y del $25,4 \%$ a $14,9 \%$, en centros privados. El análisis de estos resultados lleva a reflexionar sobre el desempeño docente en relación con sus conocimientos y sus prácticas pedagógicas. Por ello, el MINEDU viene organizando, en los últimos años, programas de capacitación docente, con el fin de implementar los cambios curriculares de manera adecuada y buscando mejorar el 
conocimiento disciplinar de los profesores. Incluso, el actual Currículo Nacional incluye ejemplos de secuencias didácticas a fin de que los docentes tengan claro el trabajo matemático que se espera que los estudiantes desarrollen, aunque dichas secuencias, en el caso de la parábola, muestran un tratamiento exclusivamente algebraico.

Como podemos ver, a pesar de los cambios curriculares que se vienen dando en Perú, la metodología de enseñanza sigue siendo tradicional, con énfasis en que los estudiantes reproduzcan procedimientos previamente realizados por ellos. Ante esto, consideramos que "el profesor es un agente crucial en los procesos de enseñanza y aprendizaje de la matemática" (Rodríguez-Flores, Picado-Alfaro, Espinoza-González \& Rojas-González, 2018, p. 90) y de allí nuestro interés por conocer el conocimiento profesional que posee.

\section{METODOLOGÍA}

\section{Protocolo de la investigación}

Este artículo presenta un instrumento que ha sido validado desde una aproximación cualitativa, diseñado en la ciudad de Lima, cuyo proceso se inició a mediados de 2018 y culminó en 2019. El protocolo seguido para su diseño consta de las siguientes etapas, descritas sintéticamente: 1) sensibilización con referentes teóricos provenientes de investigaciones con MTSK, investigaciones sobre parábola y revisión del Currículo Nacional peruano; 2) adaptación de las categorías de MTSK propuestas por Carrillo-Yañez et al. (2018) y elaboración de los indicadores para cada categoría; 3) validación de las categorías e indicadores a través de un proceso de triangulación entre investigadores internos, investigadores externos pertenecientes al grupo SIDM (Seminario de Investigación en Didáctica de la Matemática) de la Universidad de Huelva, España, y referentes teóricos; 4) reformulación de los indicadores, los cuales se muestran en la Tabla 1 de este documento; 5) formulación y validación de las preguntas del cuestionario por proceso de triangulación, en el Anexo 2 se encuentra la tabla de preguntas asociadas a cada indicador y a cada categoría; y 6) elaboración de la versión final del cuestionario (ver Anexo 1), incluyendo la reformulación de preguntas a partir de las observaciones dadas por los expertos.

Los tres expertos externos consultados para la triangulación de esta experimentación se eligieron siguiendo criterios que aseguraran su profundo conocimiento en lo que se les solicitaba asesoramiento. Así, se eligió a investigadores con publicaciones de impacto sobre el conocimiento especializado del profesor de matemáticas, cuya trayectoria investigadora les permitía aportar información relevante respecto del potencial del instrumento que aquí se presenta. Asimismo, los tres cumplen con los criterios de profesor experto planteados por Rojas, Carrillo, y Flores (2012): tienen más de cinco años de experiencia docente, han destacado en evaluaciones institucionales, son docentes recomendados por sus pares, participan en procesos de actualización de contenido (tanto matemático como de educación matemática), muestran preocupación por la mejora de los procesos educativos.

\section{Diseño del cuestionario}

Esta investigación tiene como objetivo el diseño de un instrumento de carácter cualitativo que permita explorar el conocimiento matemático del profesor con respecto a la parábola. Se eligió el cuestionario como instrumento, por su potencial como 
herramienta de obtención de información, así como por la posibilidad de que, en caso de que se decidiera escalar la investigación, pudiera ser administrado a una cantidad amplia de sujetos (Bryman, 2001). Para ello se comenzó con una sensibilización teórico-metodológica sobre estudios previos realizados con MTSK (Carrillo, Climent, Contreras y Muñoz-Catalán, 2013; Flores, Escudero y Aguilar, 2013; Flores-Medrano, Escudero-Ávila, Montes, Aguilar y Carrillo, 2014; Montes, Aguilar, Carrillo y Muñoz-Catalán, 2013; Montes, Flores Medrano, Carmona, Huitrado y Flores, 2014). Luego, se procedió a construir los indicadores para cada una de las categorías, ligados a la noción de parábola, que fueron adaptados de las categorías propuestas por Liñán, Barrera e Infante (2014), los cuales se usaron para detectar debilidades conceptuales y procedimentales en el conocimiento del profesor sobre la división entre fracciones. Para establecer los indicadores por categoría se hizo una primera adaptación de las categorías propuestas por Liñán et al. (2014), posteriormente se pasó a un proceso de triangulación, tanto al grupo de investigadores interno como al externo (Flick, 2007). Para dicha triangulación se tomó en cuenta la discusión de investigadores expertos y el análisis del Currículo Nacional (MINEDU, 2016), donde se evidencia el conocimiento que se pretende que aprendan los estudiantes sobre la parábola.

A continuación, mostramos los subdominios, las categorías y los indicadores por categoría que hemos considerado para el MK. Nótese que no todas las categorías de los subdominios están contempladas, lo que se justifica en la necesaria acotación que se preveía que debía tener el cuestionario.

Tabla 1. Indicadores sobre el conocimiento de la parábola en MTSK

\begin{tabular}{|c|c|c|}
\hline Subdominio & Categoría & Indicador \\
\hline \multirow{9}{*}{ KOT } & Definiciones & Conocimiento de la parábola como un lugar geométrico. \\
\hline & \multirow{3}{*}{ Propiedades } & $\begin{array}{l}\text { Conocimiento de la parábola como la cónica que resulta de la intersección entre } \\
\text { un cono recto y un plano paralelo a su generatriz. }\end{array}$ \\
\hline & & $\begin{array}{l}\text { Conocimiento de la propiedad de equidistancia en una parábola y de la longitud } \\
\text { del lado recto. }\end{array}$ \\
\hline & & $\begin{array}{l}\text { Conocimiento de las propiedades de simetría que presenta una parábola respecto } \\
\text { a su eje focal. }\end{array}$ \\
\hline & Fenomenología & $\begin{array}{l}\text { Conocimiento sobre la modelación de una situación cotidiana usando la defin- } \\
\text { ición de parábola como lugar geométrico. }\end{array}$ \\
\hline & \multirow[t]{2}{*}{ Registros de representación } & $\begin{array}{l}\text { Conocimiento de la representación gráfica de una parábola y su conversión a } \\
\text { una representación algebraica. }\end{array}$ \\
\hline & & $\begin{array}{l}\text { Conocimiento de la determinación de una representación algebraica para la } \\
\text { parábola a partir del reconocimiento de sus propiedades. }\end{array}$ \\
\hline & \multirow[t]{2}{*}{ Procedimientos } & $\begin{array}{l}\text { Conocimiento del procedimiento para determinar la ecuación ordinaria de una } \\
\text { parábola a partir de su ecuación general, completando cuadrados. }\end{array}$ \\
\hline & & $\begin{array}{l}\text { Conocimiento de la relación entre la ecuación ordinaria de una parábola y su } \\
\text { ecuación general, completando cuadrados. }\end{array}$ \\
\hline \multirow{3}{*}{ KSM } & \multirow[t]{2}{*}{ Conexiones de simplificación } & $\begin{array}{l}\text { Conocimiento de la relación entre la ecuación de una parábola y la regla de } \\
\text { correspondencia de una función cuadrática. }\end{array}$ \\
\hline & & Conocimiento de la relación entre parábolas y funciones cuadráticas. \\
\hline & Conexiones transversales & $\begin{array}{l}\text { Conocimiento de la relación entre parábolas, funciones cuadráticas y ecuaciones } \\
\text { cuadráticas. }\end{array}$ \\
\hline KPM & $\begin{array}{l}\text { Jerarquización y planificación } \\
\text { como forma de proceder en } \\
\text { la resolución de problemas } \\
\text { matemáticos }\end{array}$ & $\begin{array}{l}\text { Elaboración y uso de diversas estrategias en la resolución de problemas con } \\
\text { parábolas. }\end{array}$ \\
\hline
\end{tabular}

Nota: Fuente propia de la investigación. 
Tras la concreción de los indicadores para el caso de la parábola, se procedió a generar las preguntas del cuestionario, de modo que cada una de ellas permita obtener información sobre una o varias categorías. Por ejemplo, para el subdominio del conocimiento de los temas, y en particular las categorías de definición, propiedades y registros de representación, se planteó la siguiente cuestión (ver Figura 1).

En esta pregunta, nos interesa comprender cómo el profesor entiende la definición de parábola como lugar geométrico, que es la definición central, la cual se puede vincular con otros conocimientos relacionados. Para ello, el docente requiere mostrar una estrategia que permita localizar puntos equidistantes a la recta $L$ y al punto $P$, haciendo uso de algún registro de representación como el trazo de su eje de simetría, con el cual se puede determinar el vértice de la parábola, punto que geométricamente se puede hallar usando la definición de igualdad entre segmentos. De manera análoga, se pueden hallar otros puntos de la parábola con representaciones auxiliares como el trazo de un segmento desde un punto a otro punto, y el trazo de un punto a una recta dada, lo cual podría beneficiar la comprensión sobre las representaciones geométricas de distancias o de la definición de parábola. Asimismo, resulta interesante saber si el docente moviliza otras propiedades atribuibles al eje de simetría de la curva, como aquella que indica que dicho eje divide en dos mitades congruentes a la parábola y, por tal, un punto de esta curva se ve reflejado simétricamente en la otra mitad de esta misma.

En el siguiente ejemplo (Figura 2), además de centrarnos en el conocimiento de los temas en cuanto propiedades, registros de representación y procedimientos, se pretendió profundizar en el subdominio del conocimiento de la práctica matemática, respecto a la jerarquización y planificación para la resolución de problemas.

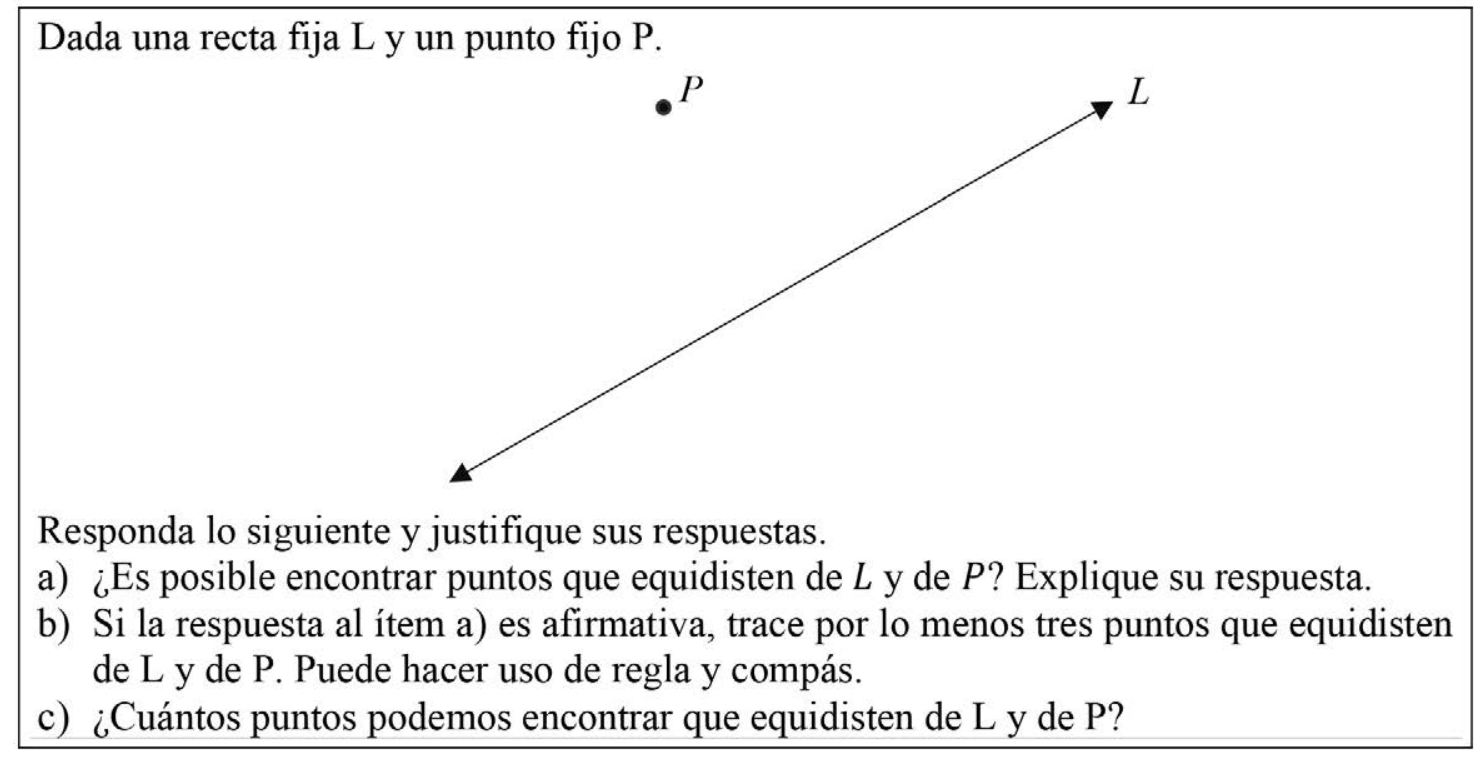

Figura 1. Pregunta 1 del cuestionario

Nota: Elaboración propia de la investigación. 
Dada una parábola con eje focal la recta $y=x$ y que pasa por el punto $P(-2 ; 4)$, determine un punto que pertenezca a dicha parábola.

Figura 2. Pregunta 4 del cuestionario

Nota: Elaboración propia de la investigación.

Esta cuestión permite indagar sobre el conocimiento que tiene el profesor respecto a algunas propiedades elementales de la parábola, como el hecho que el eje focal es el eje de simetría de la parábola. Además, nos interesa comprender cómo utiliza el conocimiento cuando el eje de simetría o eje focal de la parábola no es paralelo a los ejes del plano cartesiano. Esto requiere que se jerarquice y planifique una secuencia de resolución. Para ello el profesor debe conocer cómo determinar gráficamente el simétrico de un punto respecto a una recta, es decir, trazar una recta perpendicular al eje de simetría que pase por el punto $P$, hallar el punto de intersección $M$ entre dichas rectas y, finalmente, hallar el punto simétrico de $P$, haciendo uso de la definición de distancias iguales entre segmentos. Además, algebraicamente deberá escribir la ecuación de una recta, resolver un sistema de ecuaciones para hallar el punto de intersección y hacer uso del punto medio de un segmento.

En el ejemplo siguiente (Figura 3), se vuelve a incidir en la definición de parábola y sus propiedades, pero se pone énfasis en el papel de los registros de representación, así como en la práctica matemática de modelización, concretada en el reconocimiento de la parábola como lugar geométrico en situaciones pseudorrealistas.

Esta pregunta se propone de manera complementaria a las anteriores, y omite la referencia a los ejes coordenados. Creemos que el docente debe conocer y comprender los contenidos de la parábola (definición, propiedades y registros de representación) en situaciones no intrínsecamente matemáticas.

Cristina va a realizar una fiesta en el jardín de su casa y necesita ubicar 3 mesas con bocaditos de modo que estas queden ubicadas a la misma distancia de una mesa central (representado por un punto) y de uno de los bordes de una piscina rectangular. Determine la ubicación de las 3 mesas tomando en cuenta la ubicación mostrada para la piscina y la mesa central.
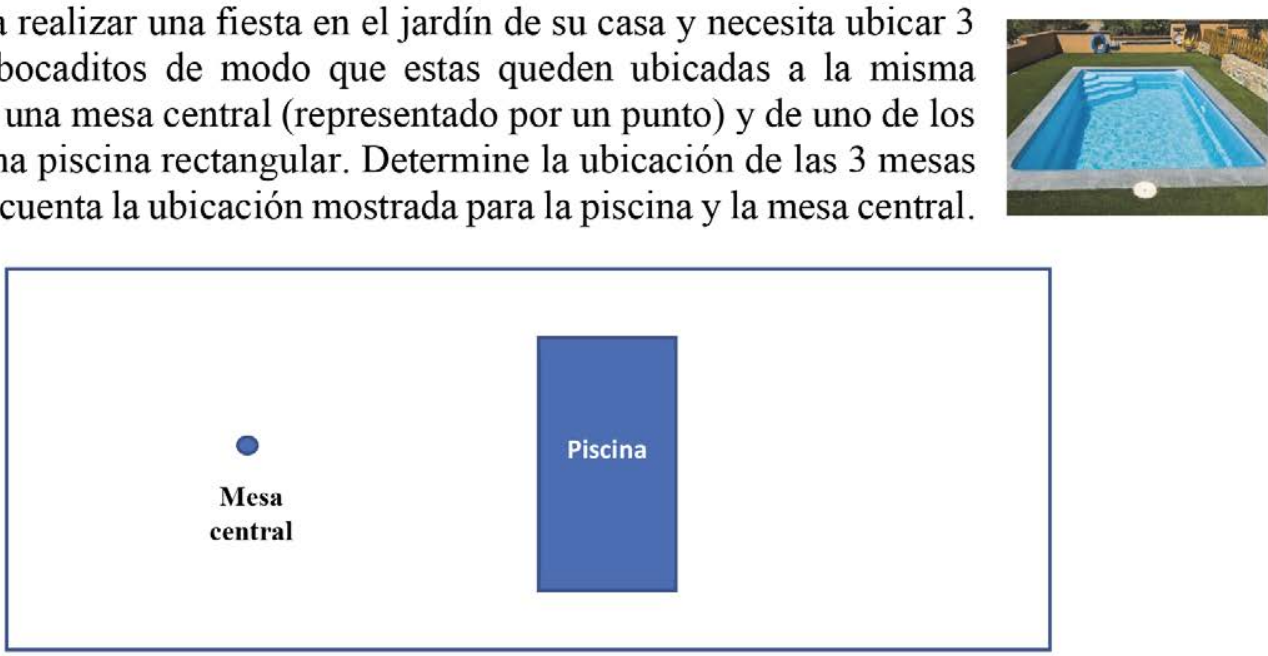

Figura 3. Pregunta 5 del cuestionario

Nota: Elaboración propia de la investigación. 
Aquí, el docente requiere desarrollar una estrategia que, basada en la definición de parábola, permita localizar, de manera gráfica, puntos equidistantes al borde de la mesa y al punto representado por la mesa. Asimismo, creemos fundamental el uso registros de representación gráfica, como el trazo del eje de simetría o el trazo de segmentos que representen la distancia desde los puntos que queremos determinar a la mesa y al borde de la piscina, ya que permiten al profesor comprender el significado de la parábola en contextos o escenarios distintos.

A continuación (Figura 4), mostramos un ejemplo de una pregunta que incide en el uso de conexiones con elementos auxiliares, como son las ecuaciones, así como en la identificación de la expresión habitual de una parábola.

Esta pregunta permite incidir en los procedimientos habituales que siguen los profesores al abordar el trabajo matemático con parábolas. Queremos conocer un procedimiento estándar de los docentes para abordar la compleción de cuadrados a partir de la ecuación general de la parábola. En este caso, profundizamos en cómo elaborar el proceso de completar cuadrados y cuándo realizar este procedimiento.

Así, se diseñó un cuestionario con un total de 20 preguntas con las que se pretende, desde su diseño, obtener información de todos los subdominios de conocimiento matemático de los profesores a los que se aplicó. Mostramos, por limitaciones de espacio, solo una síntesis del estudio de potenciales respuestas a la pregunta $1 \mathrm{del}$ cuestionario (Figura 1).
En el apartado a) se proyectaron dos potenciales respuestas:

- Respuesta 1: Un docente podría afirmar que sí es posible encontrar puntos que equidisten de $L$ y de $P$, y que dichos puntos cumplen la condición geométrica de la parábola (conjunto de puntos de un plano que son equidistantes a un punto fijo llamado foco y una recta fija llamada directriz). En este caso, el docente estaría movilizando la definición de parábola como lugar geométrico (KoT, definición).

- Respuesta 2: Un docente podría decir que es posible encontrar un solo punto, refiriéndose al punto medio del segmento $P I$, considerando que $I$ es el pie de la perpendicular trazada desde $P$ a la recta $L$. En este caso, el docente estaría dando como respuesta un caso particular, mostrando no tener interiorizada la definición de parábola (KoT, definición).

Para el apartado b) se contemplaron tres posibles respuestas:

- $\quad$ Respuesta 1: Un docente podría ubicar un punto utilizando la condición geométrica de la parábola y los instrumentos de dibujo proporcionados. Podría ubicar el vértice de la parábola, trazando una recta perpendicular a la recta $L$ que pasa por el punto $P$ y ubicando el punto $I$ que resulta de la intersección entre la recta $L$ y la perpendicular trazada, y luego ubicar el

Halle el vértice de la parábola con ecuación $2 y^{2}-12 y+x+20=0$.

Figura 4. Pregunta 9 del cuestionario

Nota: Elaboración propia de la investigación. 
punto medio del segmento PI. Podría ubicar otro punto que sería el punto de intersección $Q$ que resulta al trazar una recta paralela a la recta $L$ que pasa por el punto $P$ y un arco de circunferencia con centro en $P$ y radio $P I$. Finalmente, podría ubicar otro punto que podría ser el punto $R$, punto simétrico de $Q$ respecto del punto $\mathrm{P}$ (simetría respecto a un punto) o punto simétrico de $Q$ respecto a la recta perpendicular a $L$ que pasa por $P$ (simetría respecto a una recta). En este caso, el docente daría indicios de que conoce la definición de parábola como lugar geométrico, realizando trazos que le permiten obtener los tres puntos, usando la condición de equidistancia (KoT, propiedades), simetría de un punto respeto a otro punto o de un punto respecto a una recta (KoT, propiedades, sistemas de representación).

- Respuesta 2: Un docente solo podría ubicar un punto, el vértice, tal como se explicó en el párrafo anterior. En este caso, el docente solo estaría mostrando un caso particular, aportando evidencias de no poseer conocimiento sobre la parábola como lugar geométrico, o de no poseer destreza en la construcción geométrica de puntos que cumplan las condiciones impuestas.

- Respuesta 3: Un docente podría ubicar el vértice de la parábola, tal como se explicó anteriormente. Luego, podría ubicar un punto $T$ sobre la recta $L$, trazar el segmento $P T$, la mediatriz relativa a $P T$ y una recta perpendicular a la recta $L$ que pase por el punto $T$, y determinar el punto de intersección $S$ entre la mediatriz relativa a $P T$ y la recta perpendicular a $L$ que pasa por $T$. Finalmente, podría determinar un punto más por simetría, el punto $U$ simétrico a $S$ respecto al eje focal trazado inicialmente. En este caso, el docente evidenciaría conocer la construcción de la parábola trazando una recta perpendicular y una mediatriz (KoT, propiedades, registros de representación).

Para la pregunta c), se asumieron, a priori, dos posibles respuestas:

- $\quad$ Respuesta 1: Un docente podría decir que se pueden obtener infinitos puntos, los cuales pertenecen a una parábola (KoT, definición, sistema de representación), podría llegar a afirmar que puede existir una relación funcional entre dos variables y discutir, si el resultado es o no una función (KSM, conexiones de complejización).

- $\quad$ Respuesta 2: Un docente podría decir que solo se pueden trazar 3 puntos. En este caso, lo habitual sería que el docente solo identificase los tres puntos más fáciles de ubicar (KoT, registros de representación).

Tras este proceso, se determinó que de la pregunta 1 podría obtenerse información del KoT, especialmente en relación con la definición, las propiedades y los registros de representación, como, potencialmente, del conocimiento de la estructura matemática. Pero, no se descartó la posibilidad de obtener información de otros subdominios no esperables. Luego, se refinó la redacción de algunas preguntas, y se dio por cerrada la versión final del cuestionario (ver Anexo 1). Detallamos, a continuación, una síntesis del 
proceso seguido para el diseño del cuestionario (ver Figura 6).

\section{REFLEXIONES FINALES Y PROYECCIÓN DE LA INVESTIGACIÓN}

Este trabajo pretende aportar transparencia sobre el proceso de génesis metodológica para desarrollar instrumentos de obtención de información en estudios sobre el conocimiento del profesor, en nuestro caso, en aquellos basados en el modelo MTSK, si bien creemos que esta afirmación es extensible a la investigación realizada desde cualquier perspectiva teórica, donde los matices del desarrollo de las herramientas metodológicas tienden a estar opacadas por otros aspectos de las investigaciones realizadas.

Respecto del proceso seguido para desarrollar el cuestionario, en primer lugar, creemos de fundamental importancia profundizar en el contenido matemático que se trata de explorar, a fin de poder obtener el mayor partido posible al cuestionario y sus posibles respuestas. Esta profundización debe contemplar tanto una sensibilización hacia los conocimientos matemáticos involucrados en la investigación, como hacia los procesos de enseñanza y aprendizaje relacionados con estos mismos. En segundo lugar, destacamos la relevancia de la triangulación en el proceso de diseño del cuestionario, tanto por parte de los investigadores que llevan a cabo la investigación, de forma que se pueda asegurar la intersubjetividad (Bryman, 2001) de lo realizado; como por parte de expertos externos en las diferentes dimensiones que se abordan, ya sean los constructos teóricos que se están abordando o en la enseñanza de estos.

En este momento, el cuestionario ya ha sido implementado, y nos encontramos en el análisis de sus resultados. Sin embargo, consideramos que resulta interesante compartir el proceso, de cara a poder ofrecer un protocolo de desarrollo de cuestionarios replicable en otras investigaciones. En próximas publicaciones ofreceremos los resultados de la aplicación del cuestionario, así como, de ser el caso, una descripción de las modificaciones que entendiéramos necesarias en el cuestionario, derivadas de su aplicación.

\section{AGRADECIMIENTOS}

Esta investigación fue financiada por el Instituto de Investigación Científica de la Universidad de Lima, así como por

\begin{tabular}{|c|c|c|}
\hline Fase & Referentes usados & Triangulación \\
\hline $\begin{array}{l}\text { Sensibilización con referentes } \\
\text { teóricos }\end{array}$ & $\begin{array}{l}\text { MTSK, investigaciones sobre } \\
\text { parábola, Currículo Nacional. }\end{array}$ & No \\
\hline $\begin{array}{l}\text { Adaptación de categorías de } \\
\text { MTSK para parábola }\end{array}$ & $\begin{array}{l}\text { MTSK, conocimiento matemáti- } \\
\text { co del investigador, Currículo } \\
\text { Nacional. }\end{array}$ & $\begin{array}{l}\text { Interna, Externa-expertos en } \\
\text { MTSK }\end{array}$ \\
\hline $\begin{array}{l}\text { Primer diseño de preguntas de } \\
\text { cuestionario }\end{array}$ & $\begin{array}{l}\text { MTSK, conocimiento matemático } \\
\text { del investigador, investigaciones } \\
\text { sobre parábola. }\end{array}$ & $\begin{array}{l}\text { Interna, Externa-expertos en } \\
\text { MTSK }\end{array}$ \\
\hline $\begin{array}{l}\text { Estudio (y análisis) de potenciales } \\
\text { respuestas }\end{array}$ & $\begin{array}{l}\text { MTSK, experiencia docente, cono- } \\
\text { cimiento matemático. }\end{array}$ & $\begin{array}{l}\text { Interna, Externa- expertos en } \\
\text { MTSK, expertos en la enseñanza } \\
\text { de la parábola }\end{array}$ \\
\hline
\end{tabular}

Figura 6. Síntesis del proceso

Nota: Elaboración propia de la investigación. 
el Centro de Investigación COIDESO de la Universidad de Huelva, y por el Ministerio de Ciencia, Innovación y Universidades, del Gobierno de España (Proyecto: RTI2018-096547-B-I00).

\section{DECLARACIÓN DE LA CONTRIBUCIÓN DE LOS AUTORES}

El porcentaje total de contribución para la conceptualización, preparación y corrección de este artículo fue el siguiente: E.A.C. $20 \%$, M.B.S. $20 \%$, J.C.L.R. $20 \%$, I.T.C $20 \%$ y M.M. $20 \%$.

\section{DECLARACIÓN DE DISPO- NIBILIDAD DE LOS DATOS}

Los datos que respaldan los resultados de este estudio serán puestos a disposición por el autor correspondiente [E.A.C.], previa solicitud razonable.

\section{REFERENCIAS}

Ball, D. (1990). Prospective elementary and secondary teachers'understanding of division. Journal Research in Mathematics Education, 21(2), 132-144. https://doi. org/10.2307/749140

Bass, H. (2017). Designing opportunities to learn mathematics theory-building practices. Educational Studies in Mathematics, 95(3), 229-244. https://doi.org/10.1007/ s10649-016-9747-y

Godino, J. D., \& Batanero, C. (1994). Significado institucional y personal de los objetos matemáticos. Recherches en Didactique des Mathèmatiques, 14(3), 325-355.

Baumert, J., \& Kunter, M. (2013). The COACTIV Model of Teachers' Professional Competence. In M. Kunter, J. Baumert, W. Blum, U. Klusmann, S. Krauss, \& M. Neubrand (Eds.), Cognitive activation in the mathematics classroom and professional competence of teachers. Results from the COACTIV project (pp. 25-48). Springer International Publishing. https://doi. org/10.1007/978-1-4614-5149-5_2

Borko, H., Eisenhart, M., Brown, C. A., Underhill, R. G., Doug, J., \& Agard, P. (1992). Learning to teach hard mathematics: Do novice teachers and their instructors give up too easily?. Journal for Research in Mathematics Education, 23(3), 194-222. https://doi. org/10.2307/749118

Bryman, A. (2001). Social Research Methods. Oxford University Press.

Carreño, E., \& Climent, N. (2019). Conocimiento especializado de futuros profesores de matemáticas de secundaria. Un estudio en torno a definiciones de cuadriláteros. PNA 14(1), 23-53.

Carrillo-Yañez, J., Climent, N., Montes, M., Contreras, L., Flores-Medrano, E., Escudero-Ávila, D., Vasco, D., Rojas, N., Flores, P., Aguilar-González, A., Ribeiro, M., \& Muñoz-Catalán, M. (2018). The mathematics teacher's specialised knowledge (MTSK) model. Research in $\mathrm{Ma}$ themtics Education, 20(3), 236-253. https://doi. org/10.1080/14794802.2018.1479981

Carrillo, J., \& Contreras, L. C. (2017). Avances, utilidades y retos del modelo MTSK. En Actas de las III Jornadas del Seminario de Investigación de Didáctica de la Matemática de la Universidad de Huelva. CGSE ediciones.

Carrillo, J., Climent, N., Contreras, L. C., \& MuñozCatalán, M.C. (2013). Determining Specialized Knowledge for Mathematics Teaching. En B. Ubuz, C. Haser, y M. A. Mariotti (Eds), Actas del CERME 8 (pp. 2985 - 2994). Middle East Tecnical University, Ankara.

Del Río, C. (2018). La parábola como objeto matemático desde el enfoque ontosemiótico en el curso de Matemáticas de grado décimo del Instituto Mistrató Risaralda [Tesis de Maestría en Enseñanza de las Matemáticas, Universidad Tecnológica de Pereira Facultad de Ciencias Básicas, Pereira, Colombia].

Escudero, D. (2015). Una caracterización del conocimiento didáctico del contenido como parte del conocimiento especializado del profesor de matemáticas de secundaria [Tesis de Doctorado. Universidad de Huelva, Huelva, España].

Espinoza-Vázquez, G., Zakaryan, D., \& Carrillo Yáñez, J. (2018). El conocimiento especializado del profesor de matemáticas en el uso de la analogía en la enseñanza del concepto de 
función. Revista Latinoamericana de Investigación en Matemática Educativa, 21(3), 301324. https://doi.org/10.12802/relime.18.2133

Flick, U. (2007). Introducción a la investigación cualitativa. Morata.

Flores-Medrano, E., Escudero-Ávila, D., Montes, M., Aguilar, A., \& Carrillo, J. (2014). Nuestra modelación del conocimiento especializado del profesor de matemáticas, el MTSK. En J. Carrillo, L. C. Contreras, N. Climent, D. Escudero-Ávila, E. Flores-Medrano, \& M. Montes (Eds.), Un marco teórico para el conocimiento especializado del profesor de matemáticas ( $\mathrm{pp}$. 57-72). Universidad de Huelva Publicaciones.

Flores, E., Escudero D. I., \& Aguilar A. (2013). Oportunidades que brindan algunos escenarios para mostrar evidencias del MTSK. En A. Berciano, G. Gutiérrez, A. Estepa y N. Climent (Eds), Investigación en Educación Matemática XVII (pp. 275 - 282). Bilbao: SEIEM.

Gómez, P., \& Carulla, C. (2000). Enseñanza sobre la función cuadrática. Universidad de los Andes, Colombia.

Gómez, P., \& Ruiz, C. (2014). Influencia del software educativo GeoGebra en el aprendizaje de las cónicas en los estudiantes de $10^{\circ}$ de la Institución Educativa Simón Araujo [Tesis de Maestría, Universidad Privada Norbert Wiener, Lima, Perú].

Guerrero, L., Flores-Medrano, E., \& Carrillo, J. (2015). Conocimiento de la enseñanza de las matemáticas del profesor cuando ejemplifica y ayuda en clase de álgebra lineal. Educación Matemática, 28(2),151-174. https://doi. org/10.24844/EM2802.06

Hill, H. C., Ball, D. L., \& Schilling, S. G. (2008). Unpacking pedagogical content knowledge of students. Journal for Research in Mathematics Education, 39, 372-400.

Hill, H. C., Sleep, L., Lewis, J.M., \& Ball, D. L. (2007). Assessing teachers'mathematical knowledge: What knowledge mattres and what evidence counts. In F.K. Lester Jr. (Ed.), Second handbook of research on mathematics teaching and learning (pp. 111 - 155). Information Age.

Just, M. A., \& Carpenter, P. A. (1985). Cognitive coordinate systems: Accounts of mental rotation and individual differences in spatial ability. Psychological Review, 92, 137-132. https://doi.org/10.1037/0033-295X.92.2.137
Lara, I. (2016). La parábola como lugar geométrico: Una formación continua de profesores de profesores basada en la teoría de registros de representación semiótica [Tesis de Maestría en Enseñanza de la Matemática. Pontificia Universidad Católica del Perú, Lima, Perú].

Leinhardt, G., \& Smith, D. A. (1985). Expertise in mathematics instruction: Subject matter knowledge. Journal of Educational Psychology, 77(3), 247-271. https://doi. org/10.1037/0022-0663.77.3.247

Liñan, M., Barrera, V., \& Infante, J. (2014). Conocimiento especializado de los estudiantes para maestro: La resolución de un problema con división de fracciones. Escuela Abierta, 17(1), 41-63. doi: 10.29257/EA17.2014.04

López-Mesa, J. H., Aldana-Bermúdez, E., \& Alonso-Arboleda, A. (2013). Análisis de la comprensión del concepto de parábola en un contexto universitario. Respuestas, 18(2), 74-79. https://doi.org/10.22463/0122820X.395

Montes, M., Contreras, L. C., Liñán, M. M., Muñoz-Catalán, M. C., Climent, N., \& Carrillo, J. (2015). Conocimiento de aritmética de futuros maestros. Debilidades y fortalezas $R e-$ vista de Educación, 367, 36-62.

Montes, M., Flores-Medrano, E., Carmona, E., Huitrado, J. L., \& Flores, P. (2014). Reflexiones sobre la naturaleza del conocimiento, las creencias y las concepciones. En J. Carrillo, L. C. Contreras, N. Climent, D. Escudero-Ávila, E. Flores-Medrano, \& M. Montes (Eds.), Un marco teórico para el conocimiento especializado del profesor de matemáticas (pp. 9-22). Universidad de Huelva Publicaciones.

Montes M., Aguilar A., Carrillo J., y Muñoz-Catalán, M. C. (2013). MTSK: from Common and Horizon Knowñedge to Knowledge of Topics and Structures. En B. Ubuz, C. Haser, y M. A. Mariotti (Eds), Actas del CERME 8 (pp. 3185 3194). Middle East Tecnical University, Ankara.

Ministerio de Educación del Perú. (2016). Currículo Nacional de la Educación Básica. http:// www.minedu.gob.pe/curriculo/pdf/programa-secundaria-17-abril.pdf

Rodríguez-Flores, A., Picado-Alfaro, M., Espinoza-González, J., \& Rojas-González, N. (2018). El conocimiento especializado de un profesor de matemáticas: Un estudio de caso sobre la enseñanza de los conceptos básicos de función. Uniciencia, 32(1), 89-107. doi. org/10.15359/ru.32-1.6 
Rojas, N., Carrillo, J., \& Flores, P. (2012). Características para identificar a profesores de matemáticas expertos. En A. Estepa, Á. Contreras, J. Deulofeu, M. C. Penalva, F. J. García y L. Ordóñez (Eds.), Investigación en Educación Matemática XVI (pp. 479 - 485). SEIEM.

Ruiz, J. (2014). Una propuesta didáctica para la enseñanza de la parábola como lugar geométrico en el grado décimo en la Institución Educativa Luis López de Mesa del Municipio de Medellín [Tesis de maestría en Enseñanza de las Ciencias Exactas y Naturales, Universidad Nacional de Colombia, Medellín].

Santa, Z., \& Jaramillo, C. (2007). Construcción de las secciones cónicas mediante el doblado de papel en el marco del modelo educativo de van Hiele. X Encuentro Colombiano de Matemáticas Educativa. Universidad de Antioquia.
Scheiner, T., Montes, M. A., Godino, J. D., Carrillo, J., \& Pino-Fan, L. R. (2019). What makes mathematics teacher knowledge specialized? O'ering alternative views. International Journal of Science and Mathematics Education, 37, 270. https://doi.org/10.1007/ s10763-017-9859-6

Shulman, L. S. (1986). Those who understand: Knowledge growth in teaching. Educational Researcher, 15(2), 4-14. doi:10.3102/0013189X015002004

Stylianides, A. J., Bieda, K. N., \& Morselli, F. (2016). Proof and argumentation in mathematics education. In A. Gutiérrez, G.C. Leder \& P. Boero (Eds.), The Second Handbook of Research on the Psychology of Mathematics Education (pp. 315-351). Sense Publishers. https://doi. org/10.1007/978-94-6300-561-6_9

El conocimiento matemático del profesor acerca de la parábola: diseño de un instrumento para investigación (Elizabeth Advíncula Clemente • Marisel Beteta Salas • José Carlos León Río • Isabel Torres Céspedes • Miguel Monte). Uniciencia is protected by Attribution-NonCommercial-NoDerivs 3.0 Unported (CC BY-NC-ND 3.0) 


\section{ANEXO 1: CUESTIONARIO}

\section{Reconociendo la parábola}

Conteste las siguientes preguntas justificando sus respuestas. Para ello puede usar los instrumentos de dibujo que se le han proporcionado.

1. Dada una recta fija L y un punto fijo P.

1. Dada una recta fija $L y$ un punto fijo $P$.

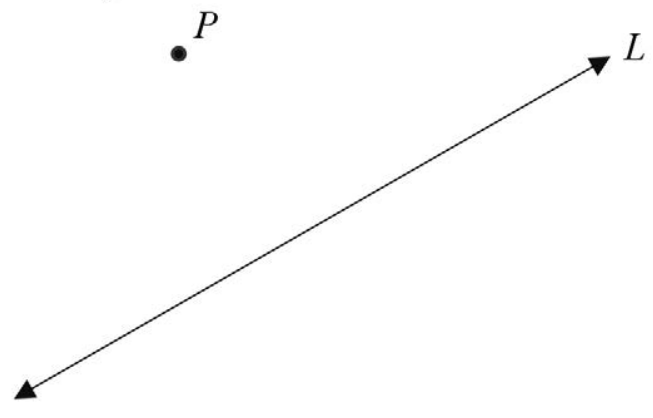

Responda lo siguiente y justifique sus respuestas.

a) ¿Es posible encontrar puntos que equidisten de L y de P? Explique.

b) Si la respuesta al ítem a) es afirmativa, trace por lo menos tres puntos que equidisten de L y de P. Puede hacer uso de regla y compás.

c) ¿Cuántos puntos podemos encontrar que equidisten de L y de P?

2. El siguiente gráfico muestra un cono de revolución.

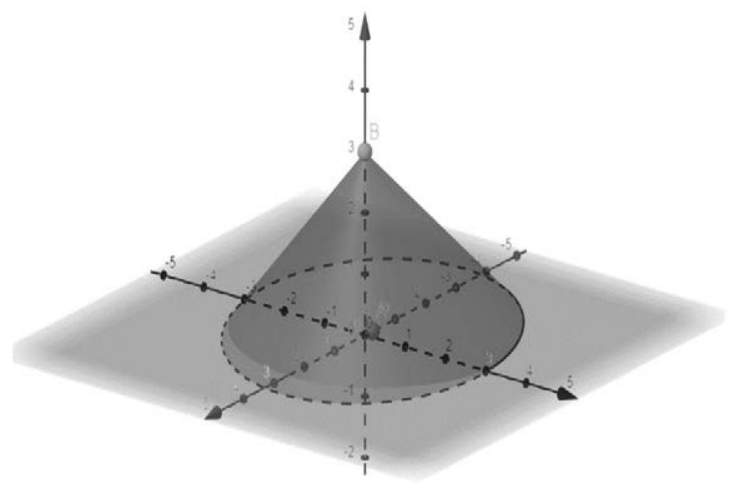

Identifique la curva que se obtiene al realizar un corte transversal paralelo a la generatriz del cono mostrado. 
3. La figura que se muestra a continuación es un rectángulo formado por dos cuadrados. Trace, de ser posible, una parábola que pase por alguno de los vértices del rectángulo. Justifique su respuesta.

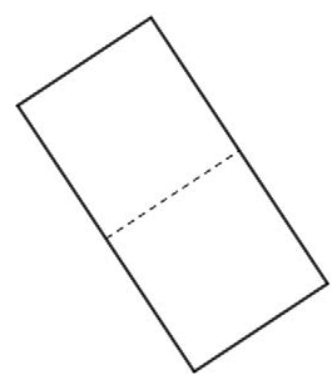

4. Dada una parábola con eje focal la recta $y=x$ y que pasa por el punto $P(-2 ; 4)$, determine un punto que pertenezca a dicha parábola.

5. Cristina va a realizar una fiesta en el jardín de su casa y necesita ubicar 3 mesas con bocaditos de modo que estas queden ubicadas a la misma distancia de una mesa central y de uno de los bordes de una piscina rectangular. Determine la ubicación de las 3 mesas tomando en cuenta la ubicación mostrada para la piscina y la mesa central.
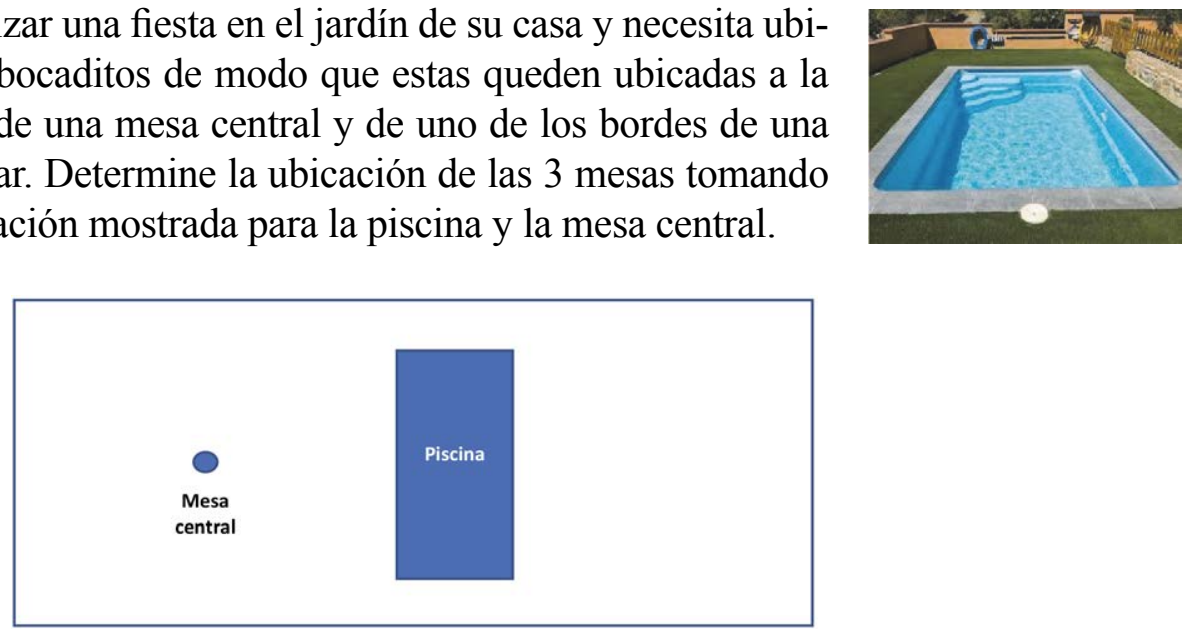

6. Una parábola se representa en el sistema de coordenadas cartesiano $R S$. En dicho sistema se indica la orientación del semieje positivo $R$ y el semieje positivo $S$. Si la distancia del vértice $V$ al eje $R$ y al eje $S$ es 2 y 4 unidades, respectivamente, y la distancia del foco $F$ al eje $R R$ y al eje $S$ es 5 y 4 unidades, respectivamente; determine la ecuación de la parábola.

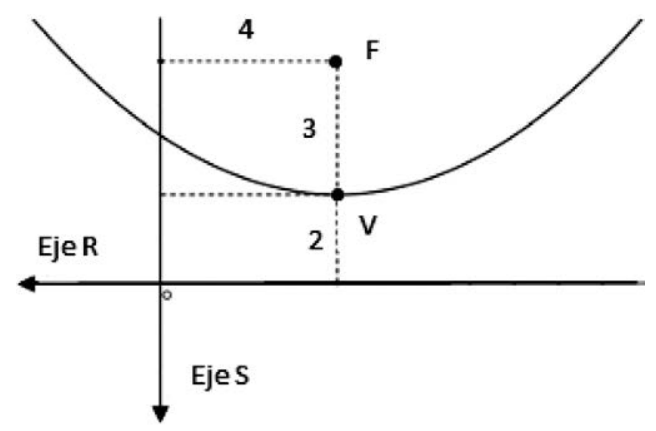


7. Halle la ecuación de la parábola con vértice en el punto $(1 ; 1)$ y directriz $x=3$.

8. Dada la ecuación de la parábola $x^{2}-8 x-10 y-4=0$, transfórmela a su forma ordinaria $\mathrm{y}$ encuentre sus principales elementos.

9. Halle el vértice de la parábola con ecuación $2 y^{2}-12 y+x+20=0$.

10. Dadas las ecuaciones siguientes: $(x-2)^{2}=-4(y+3)$

$$
(y-5)^{2}=12(x-2)
$$

¿Cuál de ellas corresponde a una función en las variables $x$ e $y$ ? Justifique su respuesta.

11. Analice el valor de verdad de las siguientes proposiciones. Justifique sus respuestas.

a) Toda parábola representa a una función cuadrática.

b) Los puntos $(-2 ; 0),(0 ; 4)$ y $(2 ; 0)$ pertenecen a la gráfica de una función cuadrática.

12. Explique la relación que existe entre el discriminante de la ecuación $a x^{2}+b x+c=0$ y el número de puntos de intersección de la gráfica de $y=a x^{2}+b x+c$ con el eje $X$. 


\section{ANEXO 2: CATEGORÍAS E INDICADORES SEGÚN MTSK Y PREGUNTAS ASOCIADAS A LA PARÁBOLA}

\begin{tabular}{|c|c|c|c|}
\hline Subdominio & Categoría & Indicador & N. ${ }^{\circ}$ pregunta \\
\hline \multirow{9}{*}{ KOT } & Definiciones & $\begin{array}{l}\text { Conocimiento de la parábola como un lugar } \\
\text { geométrico. }\end{array}$ & 1 \\
\hline & & $\begin{array}{l}\text { Conocimiento de la parábola como la cónica que } \\
\text { resulta de la intersección entre un cono recto y un } \\
\text { plano paralelo a su generatriz. }\end{array}$ & 2 \\
\hline & Propiedades & $\begin{array}{l}\text { Conocimiento de la propiedad de equidistancia en } \\
\text { una parábola y de la longitud del lado recto. }\end{array}$ & 3 \\
\hline & & $\begin{array}{l}\text { Conocimiento de las propiedades de simetría que } \\
\text { presenta una parábola respecto a su eje focal. }\end{array}$ & 4 \\
\hline & Fenomenología & $\begin{array}{l}\text { Conocimiento sobre la modelación de una situ- } \\
\text { ación cotidiana usando la definición de parábola } \\
\text { como lugar geométrico. }\end{array}$ & 5 \\
\hline & $\begin{array}{l}\text { Registros de } \\
\text { representación }\end{array}$ & $\begin{array}{l}\text { Conocimiento de la representación gráfica de una } \\
\text { parábola y su conversión a una representación } \\
\text { algebraica. }\end{array}$ & 6 \\
\hline & & $\begin{array}{l}\text { Conocimiento de la determinación de una repre- } \\
\text { sentación algebraica para la parábola a partir del } \\
\text { reconocimiento de sus propiedades. }\end{array}$ & 7 \\
\hline & Procedimientos & $\begin{array}{l}\text { Conocimiento del procedimiento para determinar } \\
\text { la ecuación ordinaria de una parábola a partir de su } \\
\text { ecuación general, completando cuadrados. }\end{array}$ & 8 \\
\hline & & $\begin{array}{l}\text { Conocimiento de la relación entre la ecuación } \\
\text { ordinaria de una parábola y su ecuación general, } \\
\text { completando cuadrados. }\end{array}$ & 9 \\
\hline \multirow{3}{*}{ KSM } & $\begin{array}{l}\text { Conexiones de } \\
\text { simplificación }\end{array}$ & $\begin{array}{l}\text { Conocimiento de la relación entre la ecuación de } \\
\text { una parábola y la regla de correspondencia de una } \\
\text { función cuadrática. }\end{array}$ & 10 \\
\hline & & $\begin{array}{l}\text { Conocimiento de la relación entre parábolas y } \\
\text { funciones cuadráticas. }\end{array}$ & 11 \\
\hline & $\begin{array}{l}\text { Conexiones } \\
\text { transversales }\end{array}$ & $\begin{array}{l}\text { Conocimiento de la relación entre parábolas, fun- } \\
\text { ciones cuadráticas y ecuaciones cuadráticas. }\end{array}$ & 12 \\
\hline KPM & $\begin{array}{l}\text { Jerarquización y } \\
\text { Planificación como } \\
\text { forma de proceder } \\
\text { en la resolución } \\
\text { de problemas } \\
\text { matemáticos }\end{array}$ & $\begin{array}{l}\text { Elaboración y uso de diversas estrategias en la } \\
\text { resolución de problemas con parábolas. }\end{array}$ & 5,6 \\
\hline
\end{tabular}

